\title{
Unit of Volume Fraction
}

National Cancer Institute

\section{Source}

National Cancer Institute. Unit of Volume Fraction. NCI Thesaurus. Code C69174.

A unit of fraction expressed as a proportion of the volume of a substance (constituent) per the volume of the system containing all constituents in the mixture under ideal conditions. 